\title{
Tethered capsule endomicroscopy: from bench to bedside at a primary care practice
}

Michalina J. Gora

Leigh H. Simmons

Lucille Quénéhervé

Catriona N. Grant

Robert W. Carruth

Weina Lu

Aubrey Tiernan

Jing Dong

Beth Walker-Corkery

Amna Soomro

Mireille Rosenberg

Joshua P. Metlay

Guillermo J. Tearney 


\title{
Tethered capsule endomicroscopy: from bench to bedside at a primary care practice
}

\author{
Michalina J. Gora, ${ }^{a, b, c}$ Leigh H. Simmons, ${ }^{\text {b,d }}$ Lucille Quénéhervé, ${ }^{\mathrm{a}, \mathrm{e}}$ Catriona N. Grant, ${ }^{\mathrm{a}}$ Robert W. Carruth, ${ }^{\mathrm{a}}$ \\ Weina Lu, ${ }^{a}$ Aubrey Tiernan, ${ }^{a}$ Jing Dong, ${ }^{\text {a,b }}$ Beth Walker-Corkery, ${ }^{d}$ Amna Soomro, ${ }^{a}$ Mireille Rosenberg, ${ }^{a, b}$ \\ Joshua P. Metlay, ${ }^{d}$ and Guillermo J. Tearney ${ }^{a, b, f, g, t}$ \\ aMassachusetts General Hospital, Wellman Center for Photomedicine, 55 Fruit Street, Boston, Massachusetts 02114, United States \\ bHarvard Medical School, 25 Shattuck Street, Boston, Massachusetts 02115, United States \\ 'Strasbourg University, CNRS, ICube Laboratory, 1 Place de l'Hopital, Strasbourg 67091, France \\ 'Massachusetts General Hospital, Division of General Internal Medicine, 55 Fruit Street, Boston, Massachusetts 02114, United States \\ eInstitut des Maladies de l'Appareil Digestif, CHU Nantes, Hôtel Dieu, place Alexis Ricordeau, Nantes 44000, France \\ fMassachusetts General Hospital, Department of Pathology, 55 Fruit Street, Boston, Massachusetts 02114, United States \\ gHarvard-MIT Division of Health Sciences Technology, 77 Massachusetts Avenue, Cambridge, Massachusetts 02139, United States
}

\begin{abstract}
Due to the relatively high cost and inconvenience of upper endoscopic biopsy and the rising incidence of esophageal adenocarcinoma, there is currently a need for an improved method for screening for Barrett's esophagus. Ideally, such a test would be applied in the primary care setting and patients referred to endoscopy if the result is suspicious for Barrett's. Tethered capsule endomicroscopy (TCE) is a recently developed technology that rapidly acquires microscopic images of the entire esophagus in unsedated subjects. Here, we present our first experience with clinical translation and feasibility of TCE in a primary care practice. The acceptance of the TCE device by the primary care clinical staff and patients shows the potential of this device to be useful as a screening tool for a broader population. ๑2016 Society of Photo-Optical Instrumentation Engineers (SPIE) [DOI: 10 .1117/1.JBO.21.10.104001]
\end{abstract}

Keywords: optical coherence tomography; digestive system; clinical translation.

Paper 160373SSR received Jun. 6, 2016; accepted for publication Sep. 12, 2016; published online Sep. 30, 2016.

\section{Introduction}

Barrett's esophagus (BE) is a condition associated with male sex, older age $(>50)$, Caucasian race, body mass index (BMI) over 30, and chronic gastroesophageal reflux disease (GERD). ${ }^{1}$ In BE, the normal squamous epithelium of the distal esophagus is replaced by columnar metaplastic epithelium. ${ }^{2}$ Specialized intestinal metaplasia is the most common type of metaplastic columnar epithelium and, though controversial, is thought to be most associated with disease progression. ${ }^{3} \mathrm{BE}$ can develop low- and high-grade dysplasia, leading to esophageal adenocarcinoma, a cancer with a poor overall 5-year survival of $15 \%$ to $20 \%$, which is growing in incidence. ${ }^{4,5}$ The risk of developing esophageal adenocarcinoma is $\sim 0.12 \%$ to $0.33 \% / y e a r$ for a patient with BE. ${ }^{6,7}$ Treatment of highgrade dysplasia and superficial adenocarcinoma is possible and curative at an early stage. ${ }^{8}$ As a result, some patients with BE risk factors are screened for BE by performing an upper endoscopy or esophagogastroduodenoscopy (EGD) with biopsies. Patients found to have histopathologic evidence of specialized intestinal metaplasia subsequently undergo regular endoscopic surveillance.

Upper endoscopy is a time-consuming and costly examination, especially in most Western countries where conscious sedation is a procedural requirement. ${ }^{9}$ In countries where the endoscopic screening is performed without sedation, this examination is not well tolerated. ${ }^{10}$ For these reasons, many people with

*Address all correspondence to: Guillermo J. Tearney, E-mail: gtearney@mgh harvard.edu
$\mathrm{BE}$ risk factors are still not screened. Considering that most $(>90 \%)$ presenting with advanced esophageal adenocarcinoma were never screened for nor therefore diagnosed with $\mathrm{BE},{ }^{11}$ there is a clear need for an improved screening tool. Such a test would have the following features: well-tolerated by patients, accurate for the detection of $\mathrm{BE}$, and inexpensive. Ideally, it would also be administered in the primary care physician (PCP) office, the central point of regular patient contact in most health care systems.

We have previously introduced a method for minimally invasive imaging of the human digestive system, termed tethered capsule endomicroscopy (TCE), ${ }^{12,13}$ that involves swallowing an engineered pill that collects cross-sectional microscopic images of the entire esophagus as it traverses the luminal organ. The capsule implements a second-generation form of optical coherence tomography (OCT) $)^{14,15}$ named optical frequency domain imaging ${ }^{16}$ or swept source OCT, ${ }^{17-19}$ capable of collecting microscopic $(10-\mu \mathrm{m}$ axial resolution) crosssectional images of the esophagus. ${ }^{20-25}$ The device comprises an $11 \mathrm{~mm}$ (diameter) $\times 24.8 \mathrm{~mm}$ (length) capsule connected to a flexible, 0.96-mm-diameter sheath, which serves as a tether (Fig. 1). The sheath encloses a driveshaft and an optical fiber; the fiber transmits light to and receives light from the miniature optics inside the capsule.

The feasibility of TCE for imaging BE has been previously published. ${ }^{12,13}$ Here, we present our experience with the technical development and clinical translation of this device to the primary care setting for BE screening.

$1083-3668 / 2016 / \$ 25.00$ @ 2016 SPIE 


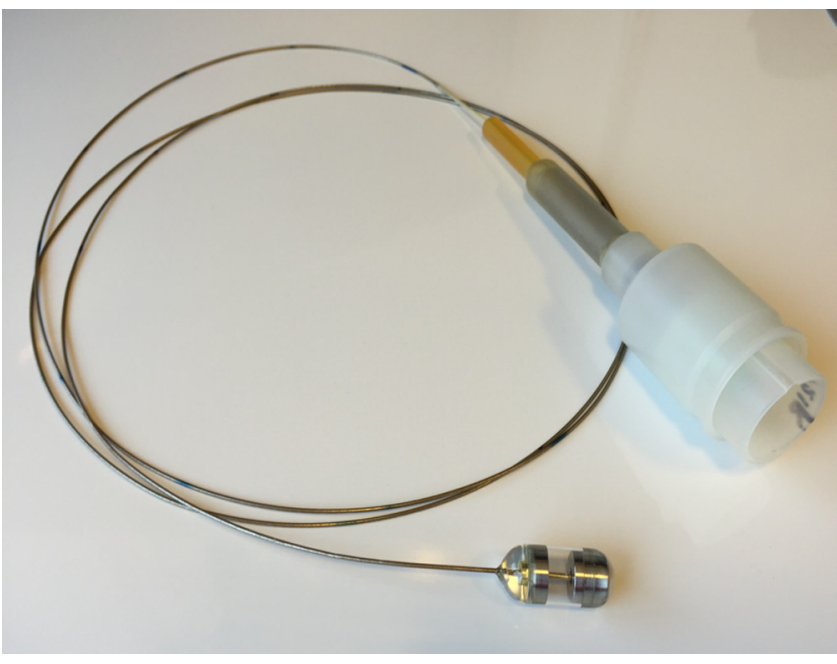

Fig. 1 TCE device comprising a capsule attached to the distal end of a flexible tether and terminated with an optical connector at the proximal end.

\section{Methods}

\subsection{Tethered Capsule Endomicroscopy Design Requirements}

The design of the TCE device was derived through an iterative process based on clinical, engineering, and regulatory requirements (Fig. 2). From a clinical use perspective, the imaging device should be minimally invasive, allowing a well-tolerated and short procedure requiring no sedation or special patient preparation. Its resolution should comprehensively, and without sampling error, enable microscopic visualization of structural

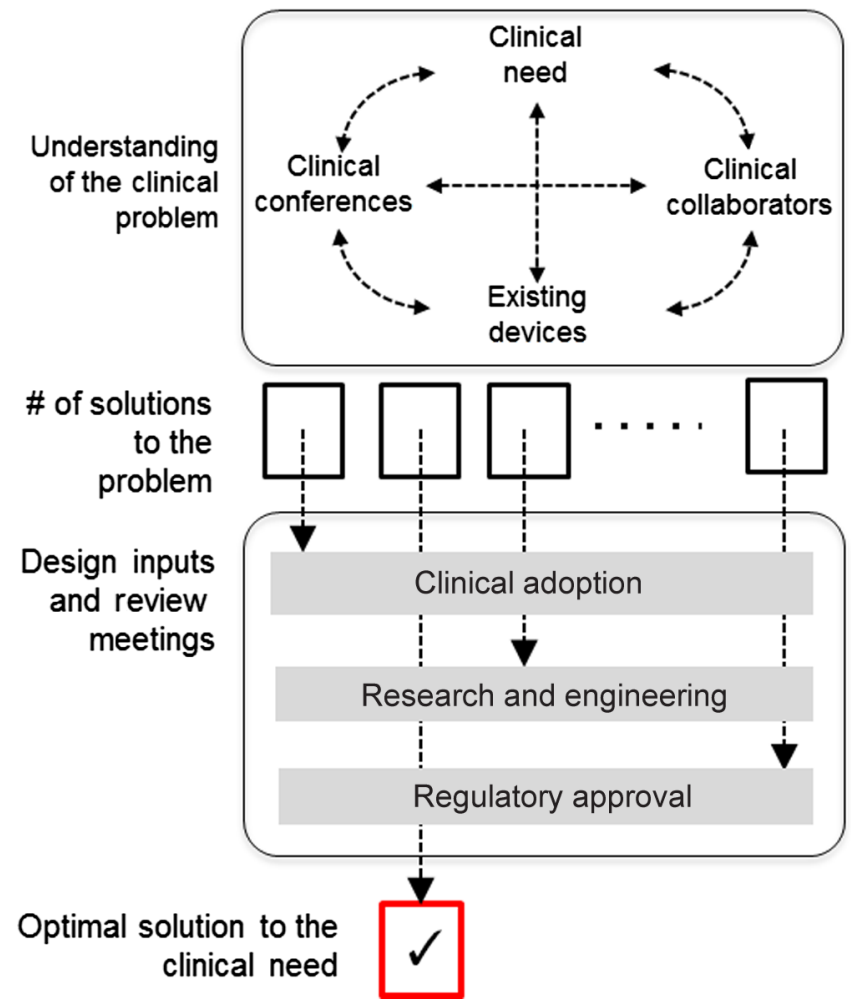

Fig. 2 Schematic representation of the device design process. changes at the level required for diagnosing the conditions of interest. Its diagnostic sensitivity should be high so as not to miss BE cases, yet also with moderate-high specificity to avoid unnecessary follow up EGDs. Its cost should be low without the specialized requirements of endoscopy suites or physicians to administer the device. Many of the engineering requirements for the device were also dictated by the nature of the digestive system (e.g., resistance to gastric acid and capability to be effectively disinfected) and were common with the design of the standard gastrointestinal (GI) endoscopes. In addition to obtaining the highest safety profile, a large effort was put into improving the ease of manufacturing the device.

\subsection{Tethered Capsule Endomicroscopy Device}

Figure 1 shows a photograph of the TCE device that was used in the primary care office. The capsule has an $11 \mathrm{~mm}$ outer diameter and a weight of $6 \mathrm{~g}$ to support natural peristalsis via gravity and improve esophageal transit. A custom multilayered sheath (microlumen) ensured flexibility while maintaining its shape, pullability, and resistance to kinking. The connection between the capsule and the sheath was covered with a flexible, tapered strain relief to improve reusability of the device, and maintain the integrity of the capsule-tether connection. A total of four catheters were manufactured for use in the PCP study; one was reused for 11 different examinations.

\subsection{Tethered Capsule Endomicroscopy Pilot Study in the Primary Care Physician's Office}

The PCP pilot study was focused on testing the safety and feasibility of TCE in primary care practice (IRB P002014-001519). The study was approved in July 2014 for imaging 20 adult patients at the Internal Medicine Associates primary care clinic at Massachusetts General Hospital (MGH) [Fig. 3(a)]. Subjects scheduled for nonurgent visits to the clinic were eligible for this study. The inclusion criteria were an age over 18 and informed consent to the study. The exclusion criteria were history of intestinal strictures, current symptoms of fever, nausea, dysphagia or sore throat, and pregnancy.

The PCP study approval was followed by a meeting of all investigators and personnel where the study design was reviewed and each participant was assigned a role in the study. The technical staff responsible for the conduct of the study introduced the device to the staff at the clinic, including the nurses and medical assistants. The PCP (L.H.S.) prescreened subjects based on the date of their scheduled appointment and their potential interest and eligibility for the study. After confirmation that all inclusion and exclusion criteria were met, subjects received a consent form and description of the study by mail and then were contacted by the research nurse over the phone. Subjects were asked to fast for $4 \mathrm{~h}$ prior to the scheduled appointment. On the day of the procedure, subjects met with the research nurse and a prequestionnaire was filled out to ensure that subjects did not have any exclusion criteria before meeting with the PCP. Fasting status was confirmed, and written consent was obtained by PCP (L.H.S.).

\subsection{Tethered Capsule Endomicroscopy Imaging Procedure}

The TCE procedure was approved to be performed before or after the scheduled PCP visit depending on the arrival of the 
Clinical procedure - total time $\sim 20 \mathrm{~min}$

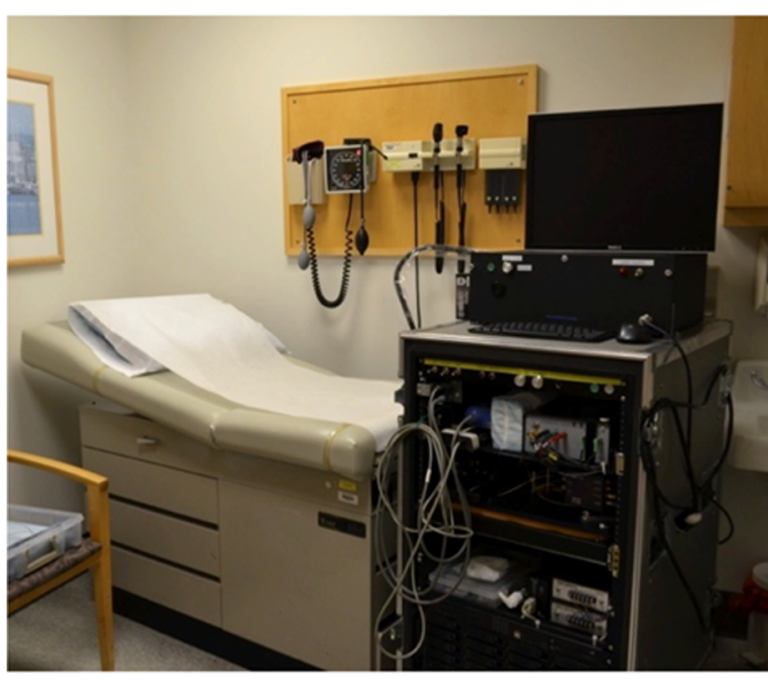

(a)

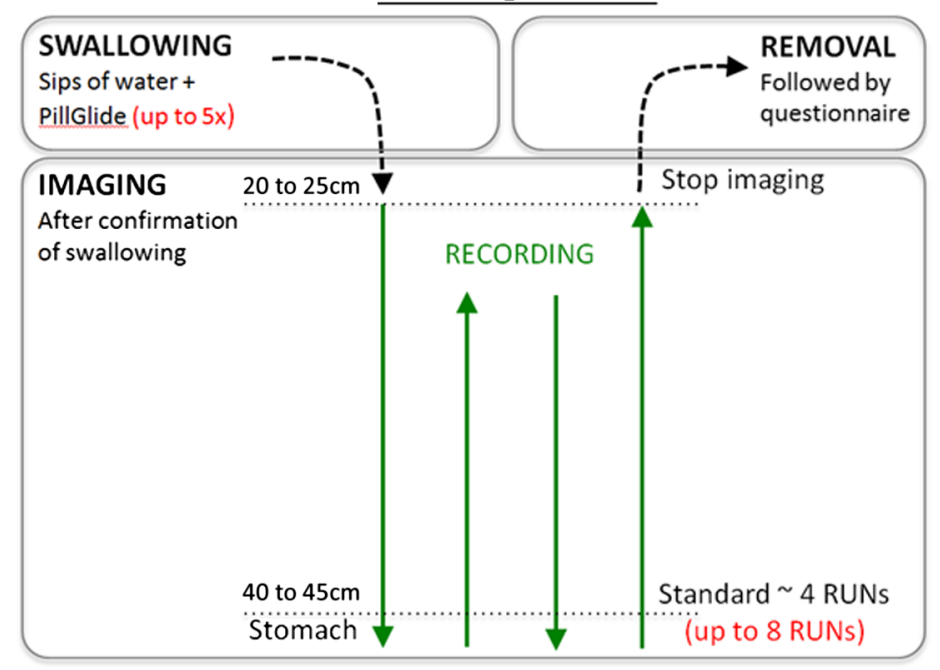

(b)

Fig. 3 (a) Photograph of the OCT imaging console in the primary care examination room and (b) schematic representation of the TCE procedure.

patient and the availability of the consenting physician. After the subject was given informed consent, clinical characteristics such as age, sex, BMI, current medication regimen, and GI history were recorded. Prior to the procedure, the subjects were invited to watch a short presentation that included a recording of prior TCE procedures and a description of the study.

Prior to study initialization, the research nurse (C.N.G.) was trained to perform all TCE procedures. Operator training comprised: reviewing recordings of TCE procedures captured during previous TCE studies performed by an experienced research nurse and a demonstration of the optimal speed for catheter navigation in the esophagus. In the next training step, the nurse performed two TCE procedures in an ongoing TCE study (IRB P0011-2619): the first one under supervision of the endoscopist with previous TCE experience and the second one without supervision. A simplified diagram of the procedure [Fig. 3(b)] was available to the nurse at all times. The nurse was accompanied by an experienced TCE imaging system operator (M.J.G.) and an additional study staff responsible for filling out case report forms (A.T.).

As shown in Fig. 3(b), the TCE clinical procedure was divided into three phases. The first swallowing phase was similar to the administration of video capsule endoscopy devices: the nonsedated subject swallowed the capsule voluntarily while sipping water. The subjects were given a maximum of five swallowing attempts. They were also encouraged to sip water to help the swallowing process. Subjects were offered the option of coating the capsule with Pill Glide ${ }^{\mathrm{TM}}$, a flavored over-thecounter lubricant spray that previously was successfully used for enhancing subjects' ability to swallow.

After the capsule was swallowed, the imaging phase began and the TCE operator navigated the device through the upper GI tract similarly to a transnasal endoscope,$^{21}$ except that the capsule's transit was governed by gravity and peristalsis until the capsule reached the stomach. Another difference was the way the device passed anatomical sphincters, such as the lower esophageal sphincter or upper esophageal sphincter during withdrawal. The natural response of these sphincters was to constrict and prevent the capsule from moving proximally from one portion of the GI tract to the other. During the pullback of the capsule using the tether, the sphincters were relaxed with a dry swallow or small sips of water taken by the subject. The study was approved for imaging the esophagus and proximal stomach up to four times. Throughout the capsule's transit, the research nurse read the capsule's position from the incisors using 5-cm marks on the tether. These readings were recorded in case report forms with corresponding TCE image frame numbers.

In order to collect OCT images of the highest quality, it was critical to ensure good contact between the lumen and the capsule wall during transit. ${ }^{22}$ If, during the imaging phase, loss of contact due to peristaltic relaxation was observed in the OCT image displayed in real-time, the catheter operator was instructed to stop the capsule by holding the tether at the incisors. The subject was then asked to dry swallow or sip water to reengage peristalsis. The dynamic response of the esophagus could be seen in the OCT data in real-time with first the expansion of the lumen, then passage of the bolus of water, and finally a constriction of the lumen around the capsule, at which point the capsule's motion was reinitiated.

The last phase of the procedure involved the removal of the capsule through the upper esophageal sphincter and the mouth. Even though sipping water efficiently relaxed the upper esophageal sphincter, it was rather cumbersome for the patient and the operator to pull the capsule out while swallowing liquid. The recommended method was to use breathing to open the sphincter. Once the capsule was out of the mouth, the total time of the procedure was recorded.

After the TCE examination, subjects were asked a set of questions:

1. How anxious did you feel before swallowing the pill? (1. not anxious to 4 . very anxious)

2. How much discomfort did you have during the procedure? (1. none to 4 . a lot) 
3. What was the hardest part of the procedure for you?

4. Would you recommend the TCE procedure to others? (1. definitely yes to 4 . definitely no).

According to the sterilization method in place at the MGH GI unit for endoscopes and manometry probes, the TCE device underwent a high level disinfection before each use at the MGH Sterile Processing Department by submersion in Cidex OPA for 12 min. After use, the TCE device was disinfected, sealed, and stored.

\section{Results}

\subsection{Subject Enrollment in the Primary Care Physician Setting}

Twenty subjects were enrolled from September 2014 to March 2015 [Fig. 4(a)]. Figure 4(b) represents the enrollment process for the study. Approximately 240 subjects were scheduled for nonurgent visits during enrollment hours, 217 were prescreened by the PCP, and 195 received information about the study by mail. Thirty-eight out of 69 subjects reached by phone were interested in participating in the study; 20 subjects were enrolled based on personnel and equipment availability. On the day of each study, the TCE system was transferred to the PCP unit and set up for the procedure in a designated outpatient examination room.

\subsection{Primary Care Physician Study Population and Study Outcomes}

Out of 20 enrolled subjects 11 were men, the average BMI was $27 \pm 4.7 \mathrm{~kg} / \mathrm{m}^{2}$, and the average age was $52 \pm 13$ years old (Table 1). After reviewing the medical files, eight patients $(40 \%)$ were found to have a history of GERD and three had already undergone an EGD for conditions other than BE (15\%).

Subjects were, on average, a little anxious before the procedure with preswallowing anxiety of $2.1 \pm 0.8$. The average number of swallow attempts was $2 \pm 1.5$. Three patients $(15 \%)$ were unable to swallow the capsule. Among the subjects who successfully swallowed the capsule, $16(94 \%)$ declared they would definitely recommend the procedure and $1(6 \%)$ said they would probably recommend it. The total TCE procedure time was $4.8 \pm 2.6 \mathrm{~min}$ with an average of $3.7 \pm 0.7$ passes in the esophagus before removal. Subjects experienced only a little discomfort $(1.9 \pm 0.9)$ during the procedures.
Table 1 Demographic characteristics and procedural tolerance in the study population.

\begin{tabular}{|c|c|}
\hline Characteristics & \\
\hline Age (year) (mean $\pm \mathrm{SD}$ ) & $52 \pm 13$ \\
\hline Men (\%) & $11(55 \%)$ \\
\hline $\mathrm{BMI}\left(\mathrm{kg} / \mathrm{m}^{2}\right)($ mean $\pm \mathrm{SD})$ & $27 \pm 4.7$ \\
\hline GERD (\%) & $8(40 \%)$ \\
\hline Race or ethnic group (\%) & \\
\hline Black or African American & $2(10 \%)$ \\
\hline Asian & $1(5 \%)$ \\
\hline Caucasian & $17(85 \%)$ \\
\hline Procedure & \\
\hline Swallowing success (\%) & $17(85 \%)$ \\
\hline Number of swallow attempts (mean \pm SD) & $2 \pm 1.5$ \\
\hline Total capsule time $(\min )($ mean $\pm \mathrm{SD})$ & $4.8 \pm 2.6$ \\
\hline $\begin{array}{l}\text { Preswallowing anxiety (mean } \pm \text { SD) } \\
\text { [(1) Not at all anxious. (2) A little anxious. } \\
\text { (3) Moderately anxious. (4) Very anxious. } \\
\text { (5) Extremely anxious.] }\end{array}$ & $2.1 \pm 0.8$ \\
\hline $\begin{array}{l}\text { Discomfort during procedure (mean } \pm \text { SD) } \\
\text { [(1) None. (2) A little. (3) Some. (4) A lot] }\end{array}$ & $1.9 \pm 0.9$ \\
\hline $\begin{array}{l}\text { Recommendation of the procedure (mean } \pm \text { SD) } \\
\text { [(1) I would definitely recommend it. (2) I would } \\
\text { probably recommend it. (3) I would probably not } \\
\text { recommend it. (4) I would definitely not } \\
\text { recommend it.] }\end{array}$ & $1.1 \pm 0.2$ \\
\hline
\end{tabular}

During the study, the research nurse introduced a new method for capsule removal based on a double breath technique, where the capsule was pulled out during the subject's second deep breath. With this method, the percentage of subjects who identified capsule removal as the most difficult part of the procedure was reduced from $42 \%$ found in a prior study ${ }^{12,13}$ to $29 \%$ in the current study.

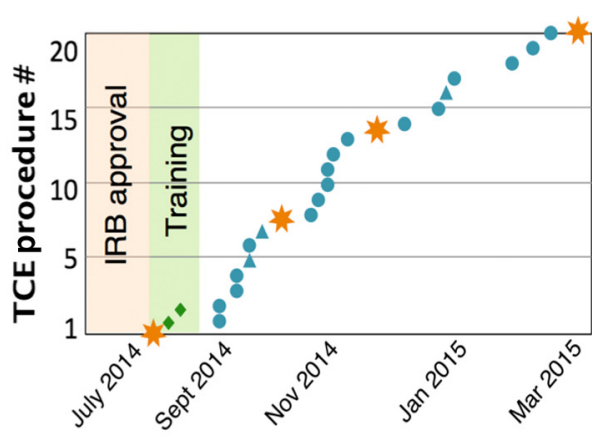

(b)

Fig. 4 (a) Patient enrollment process and (b) timeline of the PCP study. Blue circles represent successful PCP imaging procedures. Stars represent investigator meetings. Triangles correspond to procedures in which the capsule was not swallowed and diamonds correspond to training procedures. 


\subsection{Tethered Capsule Endomicroscopy Image Analysis}

The start and end positions of the capsule in the esophagus were recorded for all imaging passes based on marks on the tether. For 56 out of 61 passes, the capsule positions versus frame numbers were recorded every $5 \mathrm{~cm}$. Based on these recordings, the average length of the esophagus imaged during all studies was $15 \pm$ $6 \mathrm{~cm}$ with the average starting point in the proximal esophagus at $30 \pm 6 \mathrm{~cm}$. The average velocity of ascending imaging, determined by manual pullback of the tether, was $33.7 \mathrm{~mm} / \mathrm{s}$ with a standard deviation of $16.8 \mathrm{~mm} / \mathrm{s}$. This variability in the length of the imaged esophagus was observed even though the same research nurse performed all the procedures. The optimal pullback velocity is a tradeoff between minimal frame separation (equal to $30 \mu \mathrm{m}$ spot size), short procedure time, and ease of pulling the capsule. Based on the feedback from the catheter operator, it was difficult to perform a very slow pullback. In the interest of maintaining a short procedure duration, the optimal pullback speed was set to be $20 \mathrm{~mm} / \mathrm{s}$ corresponding to a $100-\mu \mathrm{m}$ longitudinal separation between cross-sectional TCE frames. In a majority of cases, the capsule was pulled out faster than the optimal pullback speed, providing an average frame separation for the collected 64,489 frames in 61 imaging runs of $\sim 140 \mu \mathrm{m}$.

The image signal-to-noise ratio (iSNR) was calculated for each frame by using the region in the image between the reflectance from the capsule inner and outer surfaces as the noise floor. The average iSNR of all datasets was $24.46 \pm 3.44 \mathrm{~dB}$, which indicates an adequate and reliable iSNR throughout every imaging run. Even the lowest iSNR from all 17 patients $(17.23 \mathrm{~dB})$ provided a usable dataset, as determined by expert reviewers (M.G. and G.T.). As a result, image quality was considered to be adequate for all subjects in this study.

For all subjects who swallowed the capsule, the device passed the lower esophageal sphincter and entered the stomach, allowing imaging of the gastroesophageal junction (GEJ), where $\mathrm{BE}$ can develop. A side-by-side comparison of these anatomical landmarks in two selected subjects is shown in Fig. 5. Normal features of the esophageal wall, such as a layered mucosa and a good tissue penetration depth, are displayed in Figs. 5(a) and 5(d). The proximal stomach or cardia [Figs. 5(c) and 5(f)], on the other hand, has a very poor penetration into the tissue and a typical "pit pattern" (blue arrowheads) that is visible on the surface of the tissue. The transition between these two tissues types at the GEJ is shown with a presumably normal appearance in a 36-year-old female [Fig. 5(b)] that can be compared to another transition with somewhat atypical glandular architecture in a 66-year-old female subject [Fig. 5(e)]. Similarly, irregularly shaped glands were also seen in the gastric cardia [Fig. 5(f)] of this subject. All three tissue types (squamous, GEJ, and cardia) were present in 57 out of the total of 61 passes. In three passes, only images of the esophagus were captured and in one pass, only images of the stomach were captured.

Visualization of more than $50 \%$ of the full circumference per frame was achieved in $92.3 \%$ of all 57,679 frames acquired in the esophagus and the GEJ. In every subject who swallowed the capsule, we obtained at least one imaging run where the entire circumference of the esophagus and the GEJ were clearly visualized in more than $85 \%$ of the frames. The last ascending imaging run typically had the best tissue visualization, where the full circumference of the esophagus and GEJ were clearly seen in $97 \pm 5 \%$ of the frames, and the first descending run had the lowest tissue visualization, where the full circumference
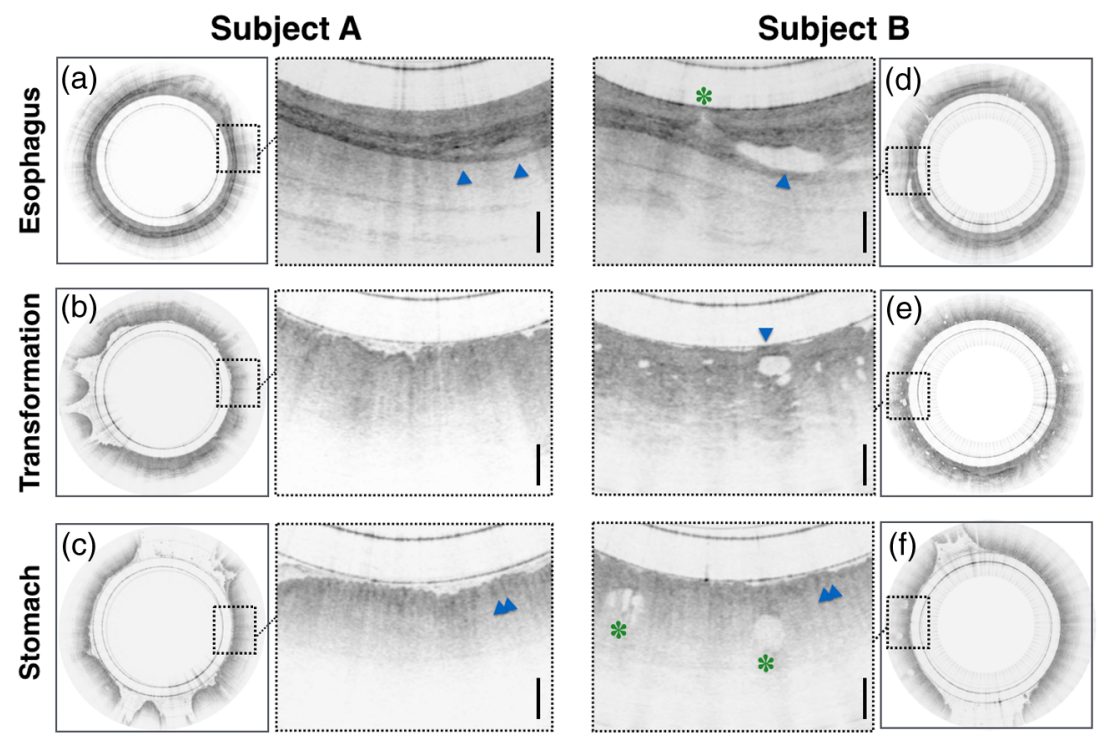

Fig. 5 Examples of TCE cross-sectional frames selected from three-dimensional data obtained in $(a, b$, c) a 36-year-old female-subject A and (d, e, f) a 66-year-old female-subject B. These images depict typical features and landmarks found in the OCT images of this study. The top row shows characteristic layered architecture of the esophageal wall with submucosal glands (blue arrowheads) in both subjects. In the example on the right (d), the submucosal gland empties into a duct that emanates at the lumen (green asterisk). The middle row shows a side-by-side comparison of the GEJ, demonstrating the transition of tissue from the squamous mucosa to the stomach's cardia, depicting a common appearance in subject $A(b)$ and an uncommon appearance characterized by the presence of glandular architecture (blue arrowhead) in subject B (d). The bottom row shows a typical pit pattern (blue arrowheads) on the surface of the gastric cardia in both subjects. In addition, subsurface glandular structures (green asterisks) can be seen below the surface of the cardia in subject $B(f)$. 
of the esophagus and GEJ were clearly visible in $86 \pm 9 \%$ of the frames.

\section{Discussion}

We have undertaken many steps to move this advanced capsulebased microscopic imaging technology, intended for screening, from the bench to the bedside to the primary care clinic. The very first TCE procedures were performed in an endoscopy suite in the MGH endoscopy unit by gastroenterologists with expertise in endoscopy. ${ }^{12}$ Following success of the first cases, the TCE procedures were moved to a regular examination room in the GI Associates Practice at MGH, where patients were seated on an examination bed. ${ }^{13}$ Based on demonstrated safety and ease of use, a trained research nurse from the GI unit was approved as the capsule operator. In this study, the technology was transferred to the primary care practice environment and the procedure was performed in a broader range of patients. This first study of TCE in the primary care population shows that this procedure is feasible in this setting, with an $85 \%$ swallowing rate, a comprehensive examination time of less than $5 \mathrm{~min}$, and the acquisition of good quality images in all cases. The procedure was successfully performed in the primary care setting that required adaptation to patients presenting without specific procedure preparation and in an exam room setting that was not designed necessarily for the performance of office procedures. Flexibility of positioning of equipment was required and was successful, e.g., in the case of a subject who used a wheelchair. The age range ( 25 to 66 years) here was also larger than that of prior TCE studies. ${ }^{12,13}$ Subject acceptability of the technique was good as demonstrated by an expression of only minor discomfort and the fact that all participants would recommend this procedure to others.

In addition, the TCE technology was well received by physicians and staff in the primary care practice. The examination was short and it was therefore possible to image patients during their time in the PCP unit without disturbing the workflow. Feedback from physicians and staff were encouraging. One of the highlighted advantages of TCE was the fact that the procedure did not require any special preparation for patients other than fasting for $4 \mathrm{~h}$. We also identified inputs to further improve the implementation of TCE in the PCP setting, including a much smaller imaging system footprint and single-user operation.

Regarding the demographics of subjects imaged in this study, it was a diverse population with a substantial rate of GERD $(40 \%)$. The majority of this cohort were white, all 11 men $(100 \%)$ were over 35 years, 5 women $(55 \%)$ were over 50 years of age, and 12 subjects $(60 \%)$ were overweight, which are all risk factors for BE. ${ }^{1}$ Only one patient had no risk factors for BE. Thus, this cohort represented a reasonable screening population for BE.

It is important to properly plan the timeline of the clinical study when translating a new technology. As presented in this paper, 20 subjects were enrolled in 16 weeks of active enrollment after contacting 102 patients by phone. Even though more subjects were interested in participating in the study, TCE device manufacturing limited the availability of the technology for enrollment. The effort in optimization of the device resulted in a technology well adapted to clinical use; however, in-house manufacturing of the device required highly trained personnel. It has to be mentioned that the transfer of a new technology to a designated environment is most commonly done after commercialization of the technology. In this study, this step was achieved with a research device. We learned that development of new solutions for decreasing the complexity of the device is necessary. Efforts were made toward solving this problem by outsourcing the manufacturing of certain subcomponent/assemblies. However, outsourcing the entire device at a reasonable cost was not feasible for our research and would limit our capabilities to further optimize the device.

The study has some limitations. Even though the goal of this study was not to measure the diagnostic accuracy of TCE for $\mathrm{BE}$, but to assess its feasibility and acceptability, a limitation was the lack of comparison with endoscopic examination and biopsy. Implementation of a validated anxiety scale would have also been useful to standardize the results and compare this new technique to others already implemented in the clinic. ${ }^{26-29}$

\section{Acknowledgments}

This work was supported in part (development of certain elements of TCE technology) by the US National Institutes of Health grants NIH R01CA103769 (G.J.T.) and 1 R01 DK10056901A1 (G.J.T.). The authors also gratefully acknowledge John and Dottie Remondi and the MGH Research Scholars program for their generous funding that enabled this work. Lucille Quénéhervé thanks the DHU 2020 (hospital and academic department-Personalized medicine for chronic diseases) of University Hospital of Nantes (France) and the SantéDige Foundation for their funding. The authors thank Katrina Armstrong from the Department of Medicine at MGH for her valuable advice and support of the study; Cynthia Ann Yeo, Kaitlyn Kelley, and Arlene Byrnes from the Internal Medicine Associate at MGH for administrative support of the study, as well as Thomas Cerruto and his team at the MGH GI unit.

\section{References}

1. L. M. Brown, S. S. Devesa, and W.-H. Chow, "Incidence of adenocarcinoma of the esophagus among white Americans by sex, stage, and age," J. Nat. Cancer Inst. 100(16), 1184-1187 (2008).

2. R. C. Haggitt, "Barrett's esophagus, dysplasia, and adenocarcinoma," Hum. Pathol. 25(10), 982-993 (1994).

3. J. Smith et al., "Intestinal metaplasia is present in most if not all patients who have undergone endoscopic mucosal resection for esophageal adenocarcinoma," Am. J. Surg. Pathol. 40(4), 537-543 (2016).

4. R. F. Souza and S. J. Spechler, "Concepts in the prevention of adenocarcinoma of the distal esophagus and proximal stomach," CA Cancer J. Clin. 55(6), 334-351 (2005).

5. W. J. Blot and J. K. McLaughlin, "The changing epidemiology of esophageal cancer," Semin. Oncol. 26(5 Suppl. 15), 2-8 (1999).

6. F. Hvid-Jensen et al., "Incidence of adenocarcinoma among patients with Barrett's esophagus," N. Engl. J. Med. 365(15), 1375-1383 (2011).

7. T. K. Desai et al., "The incidence of oesophageal adenocarcinoma in non-dysplastic Barrett's oesophagus: a meta-analysis," Gut 61(7), 970-976 (2012)

8. N. J. Shaheen et al., "ACG clinical guideline: diagnosis and management of Barrett's esophagus," Am. J. Gastroenterol. 111(1), 30-50 (2016).

9. S. J. Spechler, "Screening and surveillance for Barrett's esophagus-an unresolved dilemma," Nat. Clin. Pract. Gastroenterol. Hepatol. 4(9), 470-471 (2007).

10. M. Kruijshaar et al., "The burden of upper gastrointestinal endoscopy in patients with Barrett's esophagus," Endoscopy 38(9), 873-878 (2006).

11. G. S. Dulai et al., "Preoperative prevalence of Barrett's esophagus in esophageal adenocarcinoma: a systematic review," Gastroenterology 122(1), 26-33 (2002).

12. M. J. Gora et al., "Tethered capsule endomicroscopy enables less invasive imaging of gastrointestinal tract microstructure," Nat. Med. 19(2), 238-240 (2013). 
13. M. J. Gora et al., "Imaging the upper gastrointestinal tract in unsedated patients using tethered capsule endomicroscopy," Gastroenterology 145(4), 723-725 (2013).

14. D. Huang et al., "Optical coherence tomography," Science 254(5035), 1178-1181 (1991).

15. W. Drexler et al., "In vivo ultrahigh-resolution optical coherence tomography," Opt. Lett. 24(17), 1221-1223 (1999).

16. S. Yun et al., "High-speed optical frequency-domain imaging," Opt. Express 11(22), 2953-2963 (2003).

17. M. Choma et al., "Sensitivity advantage of swept source and Fourier domain optical coherence tomography," Opt. Express 11(18), 21832189 (2003).

18. R. Leitgeb, C. Hitzenberger, and A. Fercher, "Performance of fourier domain vs. time domain optical coherence tomography," Opt. Express 11(8), 889-894 (2003).

19. T. Wang et al., "Heartbeat OCT: in vivo intravascular megahertz-optical coherence tomography," Biomed. Opt. Express 6(12), 5021-5032 (2015).

20. M. V. Sivak et al., "High-resolution endoscopic imaging of the GI tract using optical coherence tomography," Gastrointest. Endosc. 51(4), 474-479 (2000).

21. J. M. Poneros and N. S. Nishioka, "Diagnosis of Barrett's esophagus using optical coherence tomography," Gastrointest. Endosc. Clin. N. Am. 13(2), 309-323 (2003).

22. E. Zagaynova et al., "Endoscopic OCT with forward-looking probe: clinical studies in urology and gastroenterology," J. Biophotonics 1(2), 114-128 (2008).

23. P. A. Testoni and B. Mangiavillano, "Optical coherence tomography in detection of dysplasia and cancer of the gastrointestinal tract and biliopancreatic ductal system," World J. Gastroenterol. 14(42), 6444-52 (2008).

24. T.-H. Tsai, J. G. Fujimoto, and H. Mashimo, "Endoscopic optical coherence tomography for clinical gastroenterology," Diagnostics 4(2), 57-93 (2014).

25. K. Liang et al., "Ultrahigh speed en face OCT capsule for endoscopic imaging," Biomed. Opt. Express 6(4), 1146-1163 (2015).

26. R. Likert, A Technique for the Measurement of Attitudes, Archives of Psychology (1932).

27. M. Horowitz, N. Wilner, and W. Alvarez, "Impact of event scale: a measure of subjective stress," Psychosom. Med. 41(3), 209-218 (1979).

28. C. D. Spielberger, "Manual for the state-trait anxiety inventory STAI (form Y) ("self-evaluation questionnaire")," Consulting Psychologists Press, Palo Alto, California (1983).

29. T. M. Marteau and H. Bekker, "The development of a six-item shortform of the state scale of the spielberger state-trait anxiety inventory (STAI)," Br. J. Clin. Psychol. 31(3), 301-306 (1992).

Michalina J. Gora received her $\mathrm{PhD}$ in physics from the Nicolaus Copernicus University in Poland in 2010. After that she spent 5 years at Massachusetts General Hospital (MGH) and Harvard Medical School in the laboratory of Prof. Tearney, where she developed tethered capsule OCT endomicroscopy. She is currently a researcher at the National Center for Scientific Research CNRS and ICube Laboratory in Strasbourg. She is focused on developing robotic enhanced optical diagnosing solutions for health.

Leigh H. Simmons is a general internal medicine physician who practices primary care at Massachusetts General Hospital. She conducts research in primary care practice improvement and shared decision making in routine medical care. She is on faculty at the John D. Stoeckle Center for Primary Care Innovation at MGH and is the medical director of the MGH Shared Decision Making Program.

Lucille Quénéhervé received her medical degree with a specialization in hepatology and gastroenterology in 2015 from the University Hospital of Nantes, France. She also received a master's degree in biology, biotechnology, and therapeutic research in 2014 from the University of Nantes. Currently a research fellow in the laboratory of Prof. Tearney, she conducts research on the clinical applications of OCT in gastroenterology.
Catriona N. Grant is a registered nurse who received her Bachelor of Science in Nursing degree from the University of Massachusetts Boston in 2012. She is currently a clinical research nurse in the Tearney Lab at Massachusetts General Hospital. As a member of the lab's clinical/regulatory team, her work is focused on the clinical application of the technology that is developed in the lab.

Robert W. Carruth is a senior product development engineer at DePuy Synthes in Raynham, Massachusetts. He received his BS in mechanical engineering from the Rose Hulman Institute of Technology in 2003 and his MS in biomedical engineering from the Ohio State University in 2006. He is a licensed professional engineer and worked as a senior R\&D engineer in Dr. Tearney's Lab at Massachusetts General Hospital.

Weina Lu received her BS in environmental engineering from the University of Science and Technology Beijing in China in 2009 and her MS in mechanical engineering from Worcester Polytechnic Institute in 2012. She was a R\&D engineer in Dr. Tearney's lab at the Massachusetts General Hospital from 2012 to 2015. She was involved in designing and manufacturing the catheter-based OCT endomicroscopy devices that are being used in multiple clinical trials.

Aubrey Tiernan is a gene therapy scientist at Dimension Therapeutics in Cambridge, MA, USA. She received her BS degree in chemical engineering from the University of Massachusetts, Amherst, in 2009 and her $\mathrm{PhD}$ in chemical and biomolecular engineering from the Georgia Institute of Technology in 2014. She also completed a 2-year certificate program in clinical translation at Emory University and worked as a research fellow in Dr. Tearney's lab at Massachusetts General Hospital.

Jing Dong received her $\mathrm{PhD}$ in biomedical engineering from Nanyang Technological University in Singapore in 2013. She has conducted research on developing diffuse optics-based flowmetry for noninvasive deep tissue perfusion assessment. She is currently a research fellow in Prof. Tearney's laboratory, focusing on translational research of optical coherence tomography (OCT) and its applications in gastroenterology.

\section{Beth Walker-Corkery: Biography is not available.}

Amna Soomro received her Bachelor of Medicine and Bachelor of Surgery from Dow University of Health Sciences in Karachi, Pakistan. After completing her medical boards, she became an integral part of the Wellman Center of Photomedicine, where she worked in the clinical/regulatory team in the Tearney Lab.

Mireille Rosenberg received her PhD from the Weizmann Institute in Rehovoth Israel. She has over 15 years of experience in biotechnology and has spent the last 15 years in academia in facilitation and translation of new technologies to the bedside. She is currently responsible for the regulatory and clinical team that translates the many devices developed in the Tearney laboratory to the clinic.

Joshua P. Metlay, MD, PhD, is a chief of the Division of General Internal Medicine and the Peter L. Gross chair in medicine at Massachusetts General Hospital. He is also a professor of medicine at Harvard Medical School. At MGH, he oversees an academic primary care network that includes 18 clinical sites and over 200 primary care physicians.

Guillermo J. Tearney MD, PhD, FACC, FCAP, is a professor of pathology at Harvard Medical School, the Mike and Sue Hazard Family MGH research scholar, an engineer, and a board-certified pathologist. He has conducted many of the seminal studies validating OCT and is considered an expert on OCT image interpretation. Recently, the Tearney Lab has invented a next-generation OCT technology, termed $\mu \mathrm{OCT}$. He has also pioneered tethered capsule endomicroscopy. 